OCU-PHYS-434

AP-GR-128

\title{
Charged black strings in a five-dimensional Kasner universe
}

\author{
${ }^{1}$ Hideki Ishihara*, ${ }^{2}$ Masashi Kimura ${ }^{\dagger}$ and ${ }^{1}$ Ken Matsuno ${ }^{\ddagger}$ \\ ${ }^{1}$ Department of Mathematics and Physics, \\ Osaka City University, Sumiyoshi, Osaka 558-8585, Japan \\ ${ }^{2}$ DAMTP, University of Cambridge, Centre for Mathematical Sciences, \\ Wilberforce Road, Cambridge CB3 OWA, UK
}

(Dated: June 22, 2021)

\begin{abstract}
We construct time-dependent charged black string solutions in five-dimensional Einstein-Maxwell theory. In the far region, the spacetime approaches a five-dimensional Kasner universe with an expanding three-dimensional space and a shrinking extra dimension. Near the event horizon, the spacetime is approximately static and has a smooth event horizon. We also study the motion of test particles around the black string and show the existence of quasi-circular orbits. Finally, we briefly discuss the stability of this spacetime.
\end{abstract}

PACS numbers: 04.50.-h, 04.70.Bw

\footnotetext{
* E-mail: ishihara@sci.osaka-cu.ac.jp

$\dagger$ E-mail: m.kimura@damtp.cam.ac.uk

$\ddagger$ E-mail: matsuno@sci.osaka-cu.ac.jp
} 


\section{INTRODUCTION}

Higher-dimensional spacetimes are popular subjects in fundamental theoretical physics, mainly in the context of unified theories of interactions. In order to compromise the higher dimensions with the experimentally observable four dimensions, the spacetimes would be decomposed into four-dimensional conventional spacetime in a large size and compactified extra dimensions in a small size (see ref. [1], for example, and references there in). We call the higher-dimensional spacetimes with such structures Kaluza-Klein spacetimes. The three dimensions has cosmological size while the extra dimensions should be tiny enough not to observe by experiments in laboratories. Why the large discrepancy of the sizes appears? It would be a natural idea that the extra dimensions shrink by evolution of the universe [2 [4]. If the inflation of the universe occurs by the contraction of the extra dimensions [5-7], a huge difference of the sizes can emerge.

Recently, black holes in higher dimensions gather much attention because they have rich variety in contrast to four-dimensional cases. Extended black objects, black strings for example, are found in the higher dimensions [8-11]. It is striking that exact solutions with horizon topology $S^{2} \times S^{1}$ are found [12] in addition to rotating black holes with spherical horizon topology [13] in asymptotically flat spacetimes. After this discovery, lots of related black objects were found (see ref. [14], as a review). It would be also important to investigate black hole solutions asymptote to the Kaluza-Klein spacetimes. Exact solutions which asymptote to a locally flat spacetime with a twisted $S^{1}$ extra dimension were constructed [15-22].

There is a class of exact solutions to the Einstein-Maxwell equations constructed by using harmonic functions on Ricci flat base spaces with Euclidean signature, where the metric functions and the gauge field are described by various kinds of the harmonics. If we take the harmonic function for a point source properly, the solutions describe extremely charged black holes. It is straight forward to construct multi-black hole solutions by superposition of the harmonic functions. A pioneering work was done by Majumdar and Papapetrou [23, 24] in four dimensions, and higher-dimensional solutions of this class are constructed as a variety of black holes [25 34]. In the case of hyper-Kähler base space, the solutions are supersymmetric. As generalizations of four-dimensional time-dependent multi-black hole solutions [35-37], higher-dimensional solutions including a cosmological constant [38 43] and without cosmological constant [44 48] are obtained by using harmonics. 
Indeed, exact solutions are constructed by solving the Laplace equation on a Ricci flat base space, but the solutions do not always describe black objects. If we want to construct black hole solutions by using harmonics for a point source on the Euclidean Taub-NUT space,

the point source should be on the NUT singularity for the appearance of horizon [29]. By harmonics for a line source on the four-dimensional flat Euclidean space a naked singularity appears.

In the present paper, we construct an exact solution combining a Kaluza-Klein cosmological solution and a black string solution by using a harmonic function. The solution describes a charged black string in a five-dimensional Kasner universe where three-dimensional space expands while one dimension contracts as time increases.

In the next section, the metric and the gauge field are presented, and it is shown that the spacetime has a regular event horizon where analytical extension is possible. Although the spacetime is dynamical, i.e., it has no timelike Killing vector field, the geometry near the horizon becomes static quickly in the late time, then the size of horizon does not change. This property is characterized by the existence of asymptotic Killing generator near the horizon.

In section III, we study geodesic motions of timelike and null test particles. We show the existence of quasi stable circular orbits of massive particle around the black string. The radius of the circular orbit decreases gradually, and finally falls into the horizon. Analogous to the four-dimensional Schwarzschild black hole, quasi innermost stable circular orbit appears. There also exists unstable circular orbits for massless particles.

In section $\mathrm{I}$, we discuss whether the Gregory-Laflamme instability occurs or not briefly. The section $\mathrm{V}$ is devoted to summary and discussion.

\section{BLACK STRINGS IN KALUZA-KLEIN UNIVERSE}

\section{A. Solution}

We consider time-dependent charged black strings which are exact solutions of the fivedimensional Einstein-Maxwell theory with the action

$$
S=\frac{1}{16 \pi} \int d^{5} x \sqrt{-g}\left(R-F_{\mu \nu} F^{\mu \nu}\right)
$$


The metric and the Maxwell field are given by

$$
\begin{aligned}
d s^{2} & =-H^{-2} d t^{2}+H\left[\frac{t}{t_{0}}\left(d r^{2}+r^{2} d \Omega_{\mathrm{S}^{2}}^{2}\right)+\frac{t_{0}}{t} d w^{2}\right], \\
A_{\mu} d x^{\mu} & = \pm \frac{\sqrt{3}}{2} H^{-1} d t
\end{aligned}
$$

where the function $H$ is given by

$$
H=1+\frac{M}{r},
$$

$d \Omega_{\mathrm{S}^{2}}^{2}=d \theta^{2}+\sin ^{2} \theta d \phi^{2}$ is the metric of unit two-dimensional sphere, $\mathrm{S}^{2}, t_{0}$ and $M$ are nonnegative constants. ${ }^{1}$ For the spacetime signature being $(-,+,+,+,+)$, we should require the inequality $H t / t_{0}>0$.

In the limit $M \rightarrow 0$ or $r \rightarrow+\infty$ with $t=$ finite, the field strength of the Maxwell field (3) vanishes, and the metric (2) reduces to that of the five-dimensional vacuum Kasner universe,

$$
d s^{2}=-d t^{2}+\frac{t}{t_{0}}\left(d r^{2}+r^{2} d \Omega_{\mathrm{S}^{2}}^{2}\right)+\frac{t_{0}}{t} d w^{2},
$$

which describes time evolution of an anisotropic and homogeneous universe. If the extra dimension labeled by the coordinate $w$ is compactified by periodic identification, its size becomes small enough after the evolution of the universe. The metric (2), as same as the metric (5), has a null infinity at $t=+\infty, r=+\infty$ with $r / t=$ finite.

The Kretschmann scalar is given by

$$
R^{\mu \nu \rho \sigma} R_{\mu \nu \rho \sigma}=\frac{18(r+M)^{5}\left[(r+M)^{5}-t_{0} M^{2} t r\right]+\left(t_{0} M t r\right)^{2}\left(144 r^{2}+48 M r+31 M^{2}\right)}{4 t^{4} r^{4}(r+M)^{6}} .
$$

The singularity $t=0$ with $r=$ const. corresponds to the initial cosmological singularity for the Kasner universe. Since the norm of the normal vector to a $t=$ const. surface $g^{\mu \nu}(d t)_{\mu}(d t)_{\nu}=-H^{2}$ is negative, the curvature singularity $t=0$ is spacelike. We should pay attention to a limiting surface $r \rightarrow 0$ and $t \rightarrow \infty$ keeping $r t=$ const., because the Kretschmann scalar is finite. The sizes of the two-dimensional sphere and the extra dimension become finite at the limit. As will be shown later, we find the surface is the event horizon and we extend the metric (2) across the surface. ${ }^{2}$

1 Taking the limit $N \rightarrow 0$ appears in the black hole solution in a Kaluza-Klein universe discussed in ref. [48], one can obtain the metric (2) and the Maxwell field (3). We generalize the solution (2) to multi-black string and multi-black hole solutions in Appendix $\mathrm{A}$

${ }^{2}$ As far as we consider analytic extension across the event horizon, we do not need to care about $r=-M$ singularity. 


\section{B. Analytic extension across the event horizon}

Instead of the original coordinates in (2) which do not cover $r=0$, we construct coordinates covering the surface $r=0, t=\infty$ with $r t=$ const. using a set of null geodesics. We investigate the possibility of extension by using null geodesics starting from the outer region $r>0$.

If we restrict our attention to the null geodesics confined in the $t-r$ plane, the null geodesics are determined by the null condition,

$$
-H^{-2} d t^{2}+H \frac{t}{t_{0}} d r^{2}=0
$$

namely

$$
\left(\frac{d t}{d r}\right)^{2}=\frac{t}{t_{0}}\left(\frac{M}{r}+1\right)^{3} .
$$

We use an approximate solution in the form

$$
t r=\frac{\left(2 M^{2}+u \sqrt{M r}-3 M r\right)^{2}}{4 M t_{0}}
$$

where $u$ is an arbitrary parameter. The curves (9) are approximately ingoing future null geodesics in the vicinity of $r=0$ that attain the coordinate boundary. The free parameter $u$, which labels the curves, can be used as a new coordinate. ${ }^{3}$

Now, we introduce new coordinates $(u, \rho)$ as

$$
\begin{aligned}
& r=\rho^{2} / M \\
& t=\frac{\left(2 M^{2}+(u-3 \rho) \rho\right)^{2}}{4 t_{0} \rho^{2}}
\end{aligned}
$$

3 Although we can solve the equation (8) analytically as

$$
u=2 \sqrt{t_{0} t}+(r-2 M) \sqrt{1+\frac{M}{r}}+3 M \operatorname{arcsinh}\left(\sqrt{\frac{r}{M}}\right),
$$

it is convenient to use the approximate solution (9). Eq.(9) coincides with this analytic solution up to $\mathcal{O}\left(r^{3 / 2}\right)$. 
then we rewrite the metric (2) and the Maxwell field (3) in the $(u, \rho)$ coordinates as

$$
\begin{aligned}
d s^{2}= & \left(\frac{2 M^{2}+(u-3 \rho) \rho}{2 t_{0}\left(M^{2}+\rho^{2}\right)}\right)^{2}\left[-\rho^{2} d u^{2}+2\left(2 M^{2}+3 \rho^{2}\right) d u d \rho+\rho^{2}\left(3+\frac{4 \rho^{2}}{M^{2}}\right) d \rho^{2}\right. \\
& \left.+\frac{\left(M^{2}+\rho^{2}\right)^{3}}{M^{2}} d \Omega_{\mathrm{S}^{2}}^{2}+\frac{16 t_{0}^{4}\left(M^{2}+\rho^{2}\right)^{3}}{\left(2 M^{2}+(u-3 \rho) \rho\right)^{4}} d w^{2}\right], \\
A_{\mu} d x^{\mu}= & \pm \frac{\sqrt{3} \rho}{4 t_{0}\left(M^{2}+\rho^{2}\right)}\left[\left(4 M^{2}+(u-\rho) \rho\right) d u-u \rho d \rho\right],
\end{aligned}
$$

where we omit a pure gauge term in $A_{\mu} d x^{\mu}$. The metric and the Maxwell field are regular at $\rho=0$. In the limit $\rho \rightarrow 0$ with $u=$ finite (equivalently, $r \rightarrow 0, t \rightarrow \infty$ with $\operatorname{tr}=M^{3} / t_{0}$ ), the metric (12) behaves as

$$
d s^{2} \rightarrow \frac{4 M^{2}}{t_{0}^{2}} d u d \rho+\frac{M^{4}}{t_{0}^{2}} d \Omega_{\mathrm{S}^{2}}^{2}+\frac{t_{0}^{2}}{M^{2}} d w^{2} .
$$

We also see that the $\rho=0$ surface is a null hypersurface, and the angular part of the metric, which describes $\mathrm{S}^{2} \times \mathrm{R}^{1}$, does not depend on time. Since all the metric components in (12) are analytic function of $\rho$ at $\rho=0$, the spacetime with the metric (12) gives an analytic extension of the original spacetime (2). We can see that the inner region $\rho<0$ is a time reversal of the outer region $\rho>0$ since the metric (12) is invariant under the transformation

$$
\rho \rightarrow-\rho, \quad u \rightarrow-u
$$

The outer region $\rho>0$ becomes asymptotically the Kasner universe described by (5)), then it has a future null infinity. However, any null geodesic starting from a point in the inner region cannot reach the future null infinity. Therefore, the $\rho=0$ surface is an event horizon. The exact solution (2) with (3) indeed represents the charged black string in the five-dimensional anisotropically expanding Kaluza-Klein universe.

The Penrose diagram of the spacetime with the metric (2) is shown in Fig. 1. In the outer region, the geometry looks like the five-dimensional Kasner universe described by (5) in the far region, $r \gg M$, where the three-dimensional space expands, while the compact extra dimension shrinks with the time evolution. There are a null infinity at $r=\infty, t=\infty$ and spacelike singularity at $t=0$. A null hypersurface $r \rightarrow 0, t \rightarrow \infty$ with $r t=M^{3} / t_{0}$ is the event horizon. The inner region of the event horizon is the time reversal of the outer region, and these two regions are attached with each other at the event horizon $\rho=0$. 


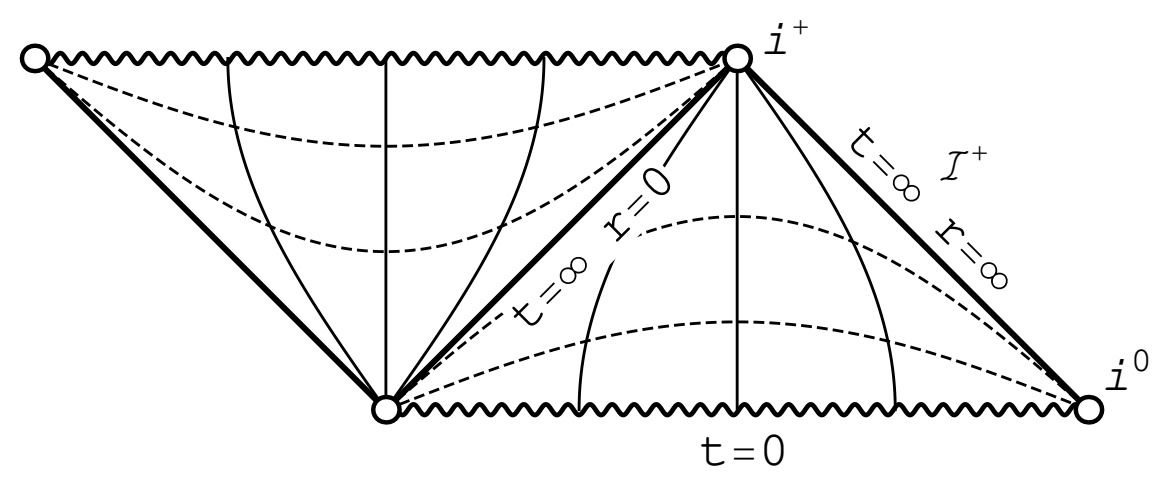

FIG. 1: Penrose diagram of $t-r$ plane. The outer region and the inner region are joined at the event horizon, $r=0$ and $t=\infty$ with $r t=M^{3} / t_{0}$. The null infinity exists at $r=+\infty$ and $t=+\infty$. The wavy lines are curvature singularities. Dashed curves denote $t=$ const. surfaces, and thin solid curves denote $r=$ const. surfaces.

\section{Approximate staticity near the event horizon}

The spacetime is dynamical because the metric (2) does not admit any timelike Killing vector. However, as shown in (14), since the metric on the horizon does not depend on time, it is suggested that the metric is approximately static near the horizon. In fact, the event horizon is an example of the 'asymptotic Killing horizon' defined in [49, 50]. In this paper, we consider an $n$-th order asymptotic Killing horizon as a generalization.

Definition. $\quad A$ null hypersurface $\mathcal{H}$ is an $n$-th order asymptotic Killing horizon if there exist a scalar function $\Phi$ and a vector field $\xi^{\mu}$, such that (i) $\Phi=0, d \Phi \neq 0$ on $\mathcal{H}$, (ii) $\mathcal{L}_{\xi^{\mu}} g_{\alpha \beta}=\mathcal{O}\left(\Phi^{n}\right), \xi^{\mu} \xi_{\mu}=\mathcal{O}(\Phi), \xi^{\mu} \nabla_{\mu} \Phi=\mathcal{O}(\Phi)$, where $\mathcal{L}_{\xi^{\mu}}$ denotes the Lie derivative with respect to a vector field $\xi^{\mu}$.

The first equation of the condition (ii) is Killing equation up to the $n$-th order of $\Phi$, and the rest of the condition (ii) requires $\xi^{\mu}$ is a generator of the horizon. We call a solution $\xi_{[n \mathrm{th}]}^{\mu} n$-th order asymptotic Killing generator. ${ }^{4}$ By choosing $\Phi=\rho$ in $(u, \rho)$ coordinate, we

\footnotetext{
4 Note that $n=1$ case is discussed in Refs. [49, 50].
} 
find a first order asymptotic Killing generator

$$
\begin{aligned}
\xi_{[1 \mathrm{st}]}^{\mu}=f & \left(\frac{\partial}{\partial u}\right)^{\mu}-\rho \partial_{u} f\left(\frac{\partial}{\partial \rho}\right)^{\mu}-\frac{2 \rho \partial_{\theta} f}{M^{2}}\left(\frac{\partial}{\partial \theta}\right)^{\mu}-\frac{2 \rho \partial_{\phi} f}{M^{2} \sin ^{2} \theta}\left(\frac{\partial}{\partial \phi}\right)^{\mu} \\
& -\frac{2 M^{4} \rho \partial_{w} f}{t_{0}^{4}}\left(\frac{\partial}{\partial w}\right)^{\mu}+\mathcal{O}\left(\rho^{2}\right),
\end{aligned}
$$

where $f$ is an arbitrary function of $u, \theta, \phi, w$. We should note that this functional degrees of freedom generally appears in the first order asymptotic Killing horizon [49, 50]. Namely, the first order asymptotic Killing generator is not determined uniquely by the geometry.

The present metric (12) also admits a second order asymptotic Killing generator

$$
\xi_{[2 \mathrm{nd}]}^{\mu}=C\left[u\left(\frac{\partial}{\partial u}\right)^{\mu}-\rho\left(\frac{\partial}{\partial \rho}\right)^{\mu}\right]+\mathcal{O}\left(\rho^{3}\right),
$$

where $C$ is an arbitrary constant. This is the unique second order asymptotic Killing generator. We understand that the second order asymptotic Killing generator $\xi_{[2 \mathrm{nd}]}^{\mu}$ characterizes approximate staticity of the geometry near the event horizon. ${ }^{5}$ Since we can show that $\xi_{\text {[2nd] }}^{\mu}$ is timelike in $\rho u>0$ and spacelike in $\rho u<0$ near the horizon, the spacetime has second order timelike asymptotic Killing generator only in the region $u>0$ outside the horizon. ${ }^{6}$ By choosing $\xi_{[2 \mathrm{nd}]}^{\mu}$ as a time coordinate basis, we can find an approximately static coordinate $(\bar{T}, \bar{R})$ in the region $u>0$ as

$$
u=e^{\bar{T} / M}(\bar{R}+M), \quad \rho=e^{-\bar{T} / M} \frac{M \bar{R}}{(\bar{R}+M)},
$$

where $\partial_{\bar{T}}=M^{-1}\left(u \partial_{u}-\rho \partial_{\rho}\right)$. In this coordinate, the metric becomes

$$
\begin{aligned}
d s^{2}=- & \frac{\bar{R}(2 M+\bar{R})^{2}(4 M+\bar{R})}{4 M^{2} t_{0}^{2}} d \bar{T}^{2}+\frac{(2 M+\bar{R})^{2}\left(2 M^{2}-2 M \bar{R}-\bar{R}^{2}\right)}{2 M(M+\bar{R}) t_{0}^{2}} d \bar{T} d \bar{R} \\
& +\frac{(2 M-\bar{R})(2 M+\bar{R})^{3}}{4(M+\bar{R})^{2} t_{0}^{2}} d \bar{R}^{2}+\frac{M^{2}(2 M+\bar{R})^{2}}{4 t_{0}^{2}} d \Omega_{\mathrm{S}^{2}}^{2} \\
& +\frac{4 t_{0}^{2}}{(2 M+\bar{R})^{2}} d w^{2}+\mathcal{O}\left(e^{-2 \bar{T} / M} \bar{R}^{2} / M^{2}\right) .
\end{aligned}
$$

We can see that the geometry near event horizon is static at the order of $\mathcal{O}(\bar{R} / M)$ as expected.

5 From the facts that the spacetime admits second order asymptotic Killing generator and the Maxwell field satisfies energy conditions, we can also show that the horizon is an isolated horizon [51].

6 Note that $\xi_{[2 \text { nd }]}^{\mu}$ has a fixed point at $u=0, \rho=0$ like a bifurcation point of a usual static black hole. 
Furthermore, we can also see that the metric near the horizon rapidly approaches the time independent form in Eq. (19) at late time $e^{-2 \bar{T} / M} \ll 1$. In fact, the time independent part in Eq. (19) can be written in a simple form

$$
d s_{\mathrm{TI}}^{2}=-\frac{R^{2}\left(R^{2}-R_{h}^{2}\right)}{R_{h}^{4}} d T^{2}+\frac{4 R^{2}}{R^{2}-R_{h}^{2}} d R^{2}+R^{2} d \Omega_{\mathrm{S}^{2}}^{2}+\frac{R_{h}^{2}}{R^{2}} d W^{2},
$$

by introducing coordinates $(T, R, W)$ as

$$
\bar{T}=\frac{1}{2} \sqrt{\frac{t_{0}}{R_{h}}} T+\frac{\sqrt{t_{0} R_{h}}}{2} \ln \frac{R-R_{h}}{\left(R+R_{h}\right)\left(2 R-R_{h}\right)^{2}}, \quad \bar{R}=\frac{2 \sqrt{t_{0}}\left(R-R_{h}\right)}{\sqrt{R_{h}}}, w=\sqrt{\frac{R_{h}}{t_{0}}} W
$$

with $R_{h}:=M^{2} / t_{0}$. The metric (20) is a limiting case of static charged black string solutions derived in ref. [10] (see Appendix B).$^{7}$

Since the time dependence appears from $\mathcal{O}\left(\bar{R}^{2} / M^{2}\right)$, we can expect the curvature tensor also has a time dependence on the horizon. While the Einstein tensor and the Kretschmann scalar take constant on the horizon, Weyl tensor has time dependence there. The leading term is same as Weyl tensor of Eq. (20) and the time dependence decays $\mathcal{O}\left(e^{-2 \bar{T} / M}\right)$ at late time.

\section{Expansion of a null geodesic congruence}

We calculate the expansions of the null vector fields emanating from a closed surface $r=$ const. on a $t=$ const. slice. The expansions are defined by

$$
\theta^{ \pm}=h^{\mu \nu} \nabla_{\mu} k_{\nu}^{( \pm)}
$$

7 Note that we can also derive Eq. (20) by taking a limit $t \rightarrow t / \epsilon, r \rightarrow \epsilon r$ and $\epsilon \rightarrow 0$ for the metric (22). In this limit the metric has the form of

$$
d s^{2}=-\frac{r^{2}}{M^{2}} d t^{2}+\frac{t M}{t_{0} r} d r^{2}+\frac{t r M}{t_{0}} d \Omega_{\mathrm{S}^{2}}^{2}+\frac{t_{0} M}{t r} d w^{2} .
$$

Though there is an apparent time dependence, by introducing coordinates

$$
R^{2}=\frac{M t r}{t_{0}}, d T=\frac{M^{2}}{t_{0} t} d t+\frac{2 M^{6} / t_{0}^{3}}{R\left(R^{2}-M^{4} / t_{0}^{2}\right)} d R, W=\frac{t_{0}}{M} w
$$

we obtain the metric form (20). 
where $k^{( \pm) \mu}$ denote future null vector fields, and $h_{\mu \nu}=g_{\mu \nu}+k_{\mu}^{(+)} k_{\nu}^{(-)}+k_{\mu}^{(-)} k_{\nu}^{(+)}$. We choose $k^{( \pm) \mu}$ as

$$
\begin{aligned}
k^{(+) \mu} \frac{\partial}{\partial x^{\mu}} & =\sqrt{\frac{t}{2 t_{0}}} \frac{\partial}{\partial t}+\frac{1}{H \sqrt{2 H}} \frac{\partial}{\partial r} \\
k^{(-) \mu} \frac{\partial}{\partial x^{\mu}} & =H^{2} \sqrt{\frac{t_{0}}{2 t}} \frac{\partial}{\partial t}-\frac{t_{0}}{t} \sqrt{\frac{H}{2}} \frac{\partial}{\partial r}
\end{aligned}
$$

such that the direction of the vector fields are to be increasing $r$ coordinates for $(+)$, and decreasing for $(-)$. Since $k^{( \pm) \mu}$ satisfy the relations $k^{(-) \mu} \nabla_{\mu} k^{(-) \nu}=0$ and $g_{\mu \nu} k^{(+) \mu} k^{(-) \nu}=$ -1 , they are regular everywhere in the spacetime. Note that $k^{( \pm) \mu}$ on and inside the event horizon should be understood as analytic extensions of them across the horizon. The metric on $\mathrm{S}^{2} \times \mathrm{R}^{1}(r=$ const., $t=$ const. $), h_{\mu \nu}$, becomes

$$
h_{\mu \nu} d x^{\mu} d x^{\nu}=H \frac{t}{t_{0}} r^{2} d \Omega_{\mathrm{S}^{2}}^{2}+H \frac{t_{0}}{t} d w^{2} .
$$

The expansions of the null geodesic congruences on the three-dimensional space (27) are obtained as

$$
\begin{aligned}
& \theta^{+}=\frac{r^{3} H^{3}+r(4 r+M) \sqrt{t_{0} t H}}{2 r^{3} H^{3} \sqrt{2 t_{0} t}} \\
& \theta^{-}=\frac{r^{3} H^{3}-r(4 r+M) \sqrt{t_{0} t H}}{2 t r^{3} H \sqrt{2 t / t_{0}}} .
\end{aligned}
$$

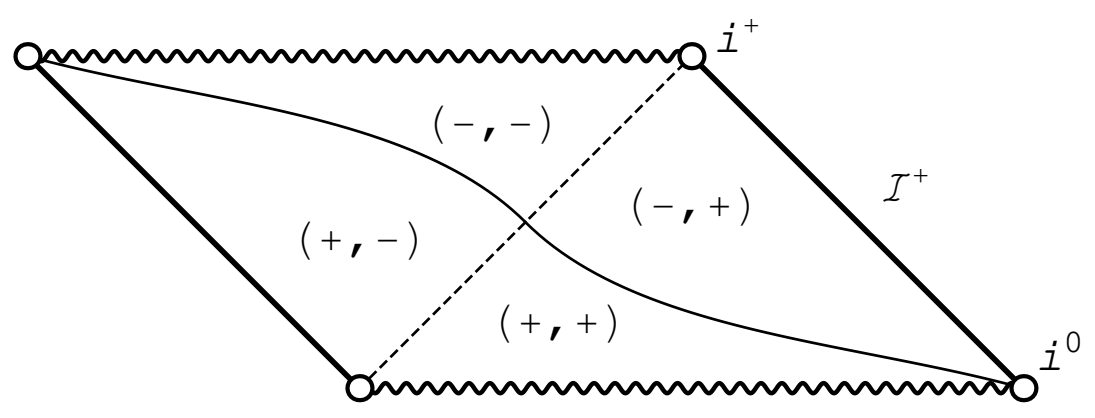

FIG. 2: The sign of $\theta^{ \pm}$and $\theta^{ \pm}=0$ surfaces in the Penrose diagram. Pairs of $( \pm, \pm)$ denote the sign of $\left(\theta^{-}, \theta^{+}\right)$. A broken line and a solid curve denote the $\theta^{+}=0$ and $\theta^{-}=0$ surfaces, respectively.

We show the $\operatorname{sign}$ of $\theta^{ \pm}$and $\theta^{ \pm}=0$ surfaces in Fig. 2, Since $\theta^{+}=0$ in the limit $r \rightarrow 0, t \rightarrow \infty$ with $t r=M^{3} / t_{0}$, we see that, though the spacetime is dynamical, the event 
horizon, $r=0, t=\infty$ with $t r=M^{3} / t_{0}$ surface, is an apparent horizon. We also see that outside the black string, $r>0, \theta^{+}$is positive, and a region $\left(\theta^{-}, \theta^{+}\right)=(+,+)$appears near the initial singularity like an expanding universe. Inside the black string, there is a trapped region, $\left(\theta^{-}, \theta^{+}\right)=(-,-)$, like a static black hole spacetime.

Using $(u, \rho)$ coordinates (10) and (11) which cover the event horizon, we can easily see the behavior of $\theta^{ \pm}$near the horizon as $\theta^{+}=\rho /\left(\sqrt{2} M^{2}\right)+\mathcal{O}\left(\rho^{2}\right)$ and $\theta^{-}=-u t_{0}^{2} /\left(4 \sqrt{2} M^{4}\right)+\mathcal{O}(\rho)$. The sign of $\theta^{+}$changes on $\rho=0$ surface, and the sign of $\theta^{-}$does on the $u=0$ surface near the horizon. Both expansions become zero at the point $(u, \rho)=(0,0)$ which is a fixed point of $\xi_{[2 \mathrm{nd}]}^{\mu}$ on the horizon.

\section{MOTION OF A TEST PARTICLE}

A test particle provides us geometrical information of a spacetime as a probe, then we study motion of it in the present spacetime. Motions of a test particle in the metric (2) is governed by the Lagrangian

$$
\mathcal{L}=\frac{1}{2}\left[-H(r)^{-2} \dot{t}^{2}+H(r) a(t)^{2}\left(\dot{r}^{2}+r^{2} \dot{\theta}^{2}+r^{2} \sin ^{2} \theta \dot{\phi}^{2}\right)+H(r) a(t)^{-2} \dot{w}^{2}\right],
$$

where dot denotes derivative with respect to the proper time of the particle, and the function $a(t)^{2}=t / t_{0}$ plays the role of cosmological scale factor for the expanding three dimensions. The particle has conserved quantities

$$
L=H a^{2} r^{2} \sin ^{2} \theta \dot{\phi}, \quad \text { and } \quad p_{w}=H a^{-2} \dot{w}
$$

Since the spacetime has the spherical symmetry, we restrict that the particle moves in the plane $\theta=\pi / 2$ without loss of generality.

The Euler-Lagrange equations for (30) with $\theta=\pi / 2$ yield

$$
\begin{aligned}
& -\frac{d}{d \tau}\left(H^{-2} \dot{t}\right)=a \frac{d a}{d t}\left(H \dot{r}^{2}+\frac{1}{H a^{4} r^{2}} L^{2}-H^{-1} p_{w}^{2}\right) \\
& \frac{d}{d \tau}\left(H a^{2} \dot{r}\right)=H^{-3} \frac{d H}{d r} \dot{t}^{2}+\frac{1}{2} \frac{d H}{d r}\left(a^{2} \dot{r}^{2}+\frac{L^{2}}{H^{2} a^{2} r^{2}}\right)+\frac{L^{2}}{H a^{2} r^{3}}+\frac{1}{2} \frac{d H}{d r} \frac{a^{2} p_{w}^{2}}{H^{2}}
\end{aligned}
$$

For a massive particle, we have

$$
g_{\mu \nu} \dot{x}^{\mu} \dot{x}^{\nu}=-H^{-2} \dot{t}^{2}+H a^{2} \dot{r}^{2}+\frac{L^{2}}{H a^{2} r^{2}}+\frac{a^{2}}{H} p_{w}^{2}=-1 .
$$




\section{A. Quasi circular orbits}

We should note that the canonical momentum conjugate to the time $t$,

$$
p_{t}=\frac{\partial \mathcal{L}}{\partial \dot{t}}=-H^{-2} \dot{t}
$$

is not conserved since the metric depends on $t$ through the function $a(t)$. This is because

the metric describes a black string residing in an evolving universe. In a much late stage of the universe, say $t=t_{1}$, the time scale of the evolution,

$$
a(t)\left(\frac{d a(t)}{d t}\right)^{-1}=2 t_{1}
$$

becomes much longer than the time scale of an orbiting particle near the black string. For this particle, $a(t)$ is almost constant at $t \sim t_{1}$ during its typical motion, then $p_{t}$ is almost constant, say $-E$. In this case, (33) is rewritten as

$$
H^{-1} a^{2} \dot{r}^{2}+U_{\text {eff }}=E^{2}
$$

where

$$
U_{\text {eff }}=H^{-2}\left(1+\frac{L^{2}}{H a^{2} r^{2}}+\frac{a^{2}}{H} p_{w}^{2}\right)
$$

then, we can analyze orbits of the particle using the effective potential (37) with a constant $a\left(t_{1}\right)$.

Here, taking the cosmological scale factor into account, we introduce a radial coordinate that denotes 'physical length' on a time-slice $t=t_{1}$ as

$$
\tilde{r}=a\left(t_{1}\right) r
$$

Using $\tilde{r}$, we represent the effective potential (37) as

$$
U_{\mathrm{eff}}\left(\tilde{r}, t_{1}\right)=\frac{\tilde{r} L^{2}+\tilde{r}^{3} a\left(t_{1}\right)^{2} p_{w}^{2}+\tilde{r}^{2}(\tilde{M}+\tilde{r})}{(\tilde{M}+\tilde{r})^{3}}
$$

where

$$
\tilde{M}=a\left(t_{1}\right) M
$$

The size of compactified dimension $w$ becomes very small owing to the evolution of the universe. It would be expected that the momentum $p_{w}$ conjugate to $w$ is hardly excited [52]. 


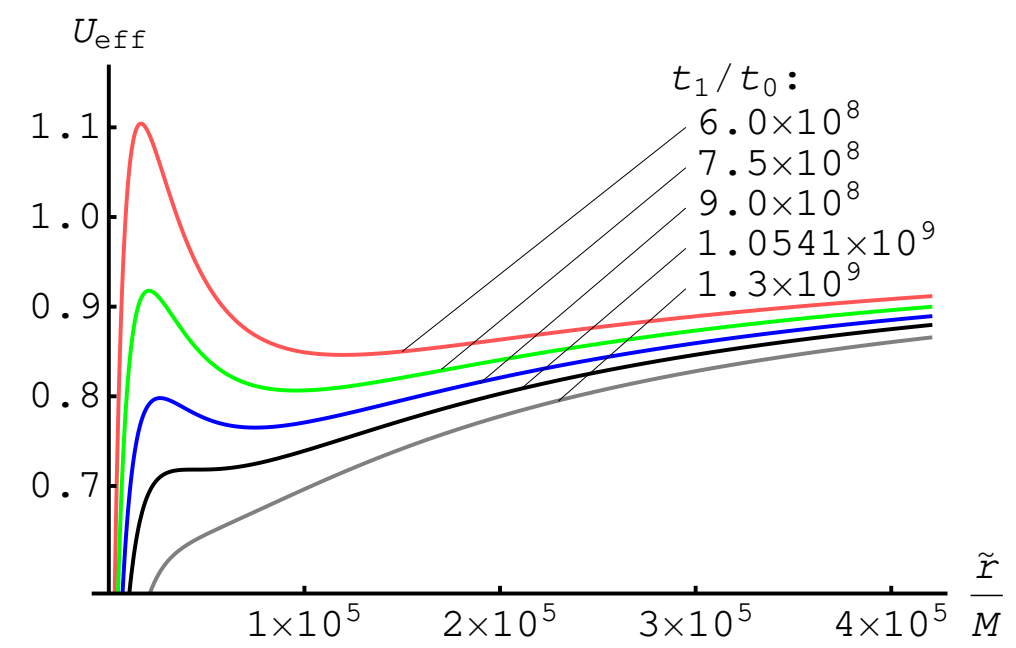

FIG. 3: The behavior of the effective potential (41) versus $\tilde{r} / M$ in various $t_{1} / t_{0}$ for $L / M=62722$. The effective potential evolves as $t_{1} / t_{0}$ increases: $t_{1} / t_{0}=6 \times 10^{8}$ (red curve), $t_{1} / t_{0}=7.5 \times 10^{8}$ (green curve), $t_{1} / t_{0}=9 \times 10^{8}$ (blue curve), $t_{1} / t_{0}=(2-\sqrt{3}) L^{2} / M^{2} \simeq 1.0541 \times 10^{9}$ (black curve), and $t_{1} / t_{0}=1.3 \times 10^{9}$ (gray curve).

Then, we consider the case $p_{w}=0$. The restriction of the metric (2) and the particle motion on $w=$ const. surface would be a model of a four-dimensional black hole in an expanding universe. In this case, (39) reduces to

$$
U_{\text {eff }}\left(\tilde{r}, t_{1}\right)=\frac{\tilde{r} L^{2}+\tilde{r}^{2}(\tilde{M}+\tilde{r})}{(\tilde{M}+\tilde{r})^{3}} .
$$

The effective potential (41) is shown as the function of $\tilde{r}$ in Fig. 3. Since $\tilde{M}$ depends on time $t_{1}$, the effective potential (41) gradually changes in its shape as the universe expands.

The existence of an innermost stable circular orbit (ISCO) is a feature of four-dimensional stationary black holes. It is an interesting question whether the ISCO exists or not in the present case. Then, we concentrate on circular orbits.

If $a\left(t_{1}\right)$ and $E$ were constants, for a fixed $L$, there exist a stable circular orbit and an unstable one specified by

$$
U_{\text {eff }}-E^{2}=0, \quad \text { and } \quad \frac{d U_{\text {eff }}}{d \tilde{r}}=0
$$

In fact, since $a\left(t_{1}\right)$ and $E$ gradually changed as time increases, the radius of the orbits specified by (42) are gradually changed. Then, we call these orbits 'quasi circular orbits'. 
The radius of the stable quasi circular orbit is

$$
\tilde{r}_{s}=\frac{1}{2 \tilde{M}}\left(L^{2}-\tilde{M}^{2}+\sqrt{\left(L^{2}-\tilde{M}^{2}\right)^{2}-2 \tilde{M}^{2} L^{2}}\right),
$$

and the radius of the unstable quasi circular orbit is

$$
\tilde{r}_{u}=\frac{1}{2 \tilde{M}}\left(L^{2}-\tilde{M}^{2}-\sqrt{\left(L^{2}-\tilde{M}^{2}\right)^{2}-2 \tilde{M}^{2} L^{2}}\right) .
$$

In order to have real positive $\tilde{r}_{s}, L^{2} \geq(2+\sqrt{3}) \tilde{M}^{2}$. In the case of $L^{2}=(2+\sqrt{3}) \tilde{M}^{2}$, we have the radius of the quasi innermost stable circular orbit (qISCO) is given by

$$
\tilde{r}_{\mathrm{qISCO}}=\frac{1+\sqrt{3}}{2} \tilde{M}
$$

and a qISCO particle has the angular momentum

$$
L= \pm L_{\mathrm{qISCO}}= \pm \frac{1+\sqrt{3}}{\sqrt{2}} \tilde{M}
$$

The ratio $r / M$ for the qISCO is not smaller than unity, then there does not exist quasi circular orbit in the approximately static region near the horizon.

Note the time dependence of $\tilde{M}$, we see that $\tilde{r}_{s}, \tilde{r}_{u}$, and $\tilde{r}_{\mathrm{qISCO}}$ are time dependent. Suppose a quasi circular particle at $\tilde{r}_{s}\left(>\tilde{r}_{\text {IISCO }}\right)$ for a fixed angular momentum $L$ at a time $t_{1}$ in the present metric with fixed parameters $M$ and $t_{0}$. As the time $t_{1}$ increases the radius $\tilde{r}_{s}$ decreases and the radius $\tilde{r}_{u}$ increases (see Fig. (4). At the time $t_{1} / t_{0}=(2-\sqrt{3}) L^{2} / M^{2}, \tilde{r}_{s}$ and $\tilde{r}_{u}$ merge together at $\tilde{r}_{\mathrm{qISCO}}$. The particle with $L$ has no circular orbit after that time, then the particle plunges into the black string.

Numerical calculations of the equations of motion (32) shows the existence of quasi circular orbits (see Fig. 51), and the particle in the orbit plunges into the black string.

The period of quasi circular orbit is given by

$$
T=2 \pi \frac{d t}{d \phi}=\sqrt{2} \pi \sqrt{\frac{(\tilde{M}+\tilde{r})^{3}(\tilde{M}+2 \tilde{r})}{\tilde{M} \tilde{r}}} .
$$

We can verify $t_{1}$ is much larger than $T$ in the numerical calculations. In the large $\tilde{r}$ limit, we have

$$
T \rightarrow 2 \pi \sqrt{\frac{\tilde{r}^{3}}{\tilde{M}}}
$$

It means the Kepler's third law. 


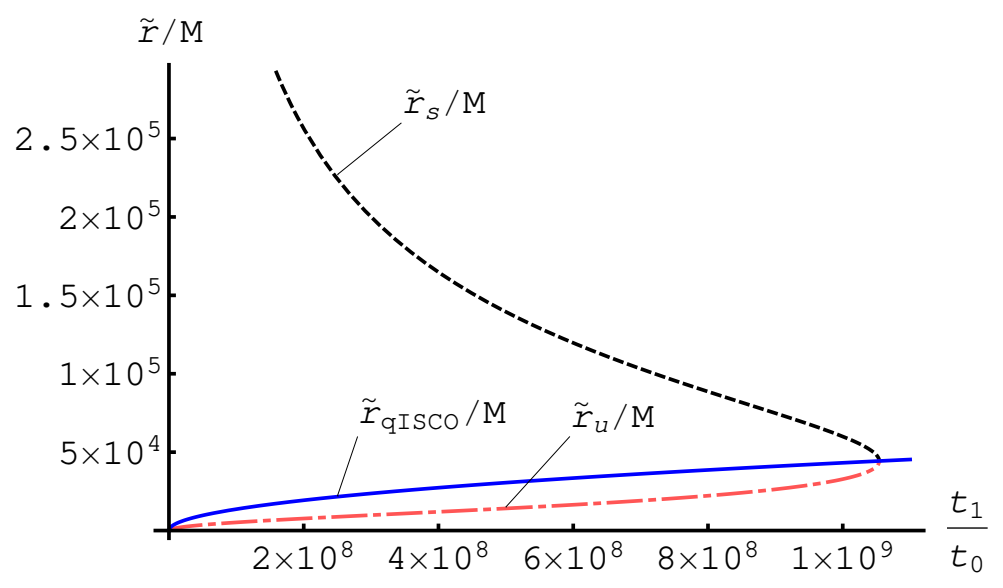

FIG. 4: The behavior of the radius of the quasi circular orbits, $\tilde{r}_{s} / M$ (black broken curve), $\tilde{r}_{u} / M$ (red dashed-dotted curve), and $\tilde{r}_{\mathrm{qISCO}} / M$ (blue solid curve), versus $t_{1} / t_{0}$ for $L / M=62722$. At the time $t_{1} / t_{0}=(2-\sqrt{3}) L^{2} / M^{2} \simeq 1.0541 \times 10^{9}, \tilde{r}_{s}$ and $\tilde{r}_{u}$ merge together at $\tilde{r}_{\mathrm{qISCO}}$.

\section{B. Massless particles with $p_{w}=0$}

Here, we consider a massless particle with $p_{w}=0$. We consider the four-dimensional metric

$$
d s^{2}=a(\eta)^{2}\left[-H^{-2} d \eta^{2}+H\left(d r^{2}+r^{2} d \Omega_{\mathrm{S}^{2}}^{2}\right)\right]
$$

where we have used the conformal time $\eta=2 \sqrt{t t_{0}}$. Since a null geodesic is invariant under a conformal transformation, we can consider a null geodesic in the static metric

$$
d \bar{s}^{2}=-H^{-2} d \eta^{2}+H\left(d r^{2}+r^{2} d \Omega_{\mathrm{S}^{2}}^{2}\right)
$$

Since we can set $\theta=\pi / 2$ without loss of generality, the Lagrangian for the massless particle in this metric is

$$
\mathcal{L}=\frac{1}{2}\left(-H^{-2} \dot{\eta}^{2}+H\left(\dot{r}^{2}+r^{2} \dot{\phi}^{2}\right)\right)
$$

where the over dot denotes derivative with respect to an affine parameter. Conserved quantities are

$$
E=H^{-2} \dot{\eta}, \quad L=H r^{2} \dot{\phi}
$$

which are exactly conserved for massless particles. The massless condition is

$$
H^{-1} \dot{r}^{2}+U_{\text {eff }}=E^{2}
$$




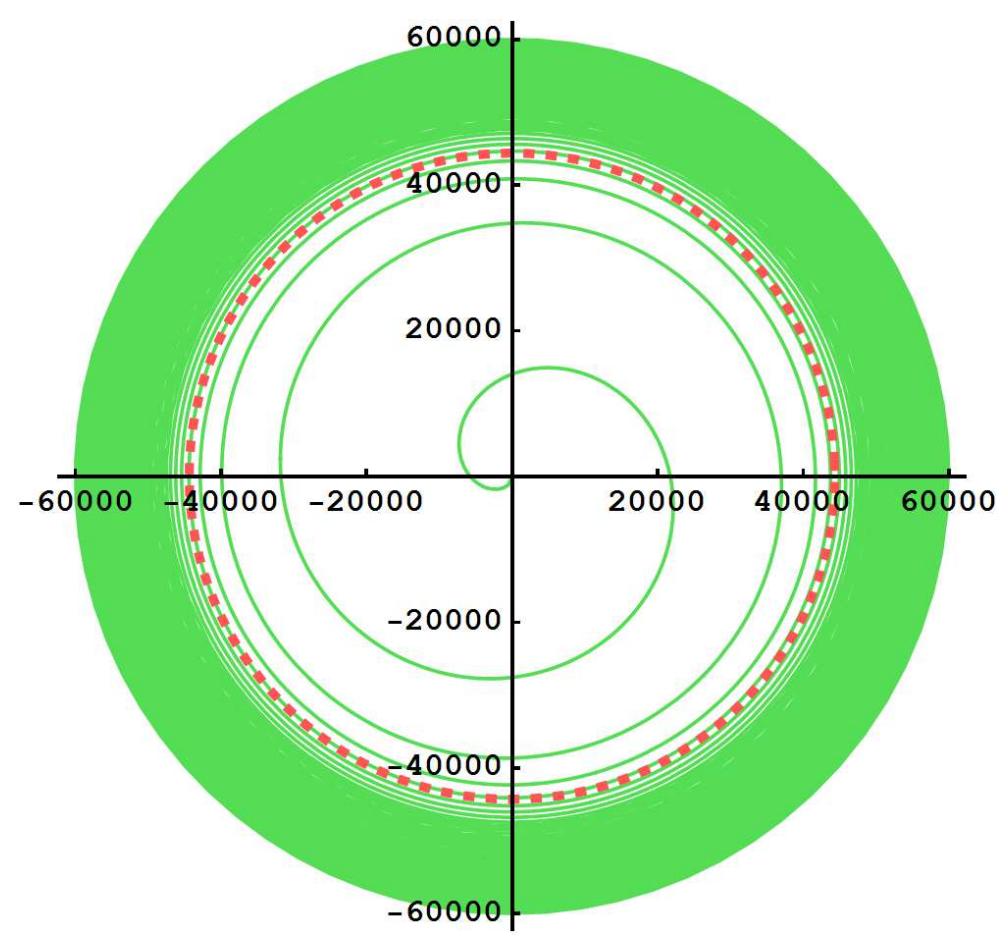

FIG. 5: Numerical calculation of a quasi stable circular orbit of a massive particle with $L / M=$ 62722 in the $\tilde{r}-\phi$ plane. We set the initial radius of the circular orbit as $\tilde{r}_{s} / M=60000$ at $t_{1} / t_{0}=10^{9}$. In this set up, $T / t_{0}=1.1015 \times 10^{6}$, then the condition $t_{1} \gg T$ holds. The radius $\tilde{r}_{s}$ and the period $T$ decreases gradually as the time $t_{1}$ increases. At the time $t_{1} / t_{0}=(2-\sqrt{3}) L^{2} / M^{2} \simeq$ $1.0541 \times 10^{9}$, the particle arrives at $\tilde{r}_{\mathrm{qISCO}} / M=44351$ (red dotted circle), then plunges into the black string.

where

$$
U_{\text {eff }}=\frac{L^{2}}{H^{3} r^{2}}
$$

Therefore, the orbits are integrable in the case of $p_{w}=0$. By using the effective potential $U_{\text {eff }}$, it is easy to know the existence of unstable circular null orbit as same as the Schwarzschild black hole. The radius is determined by

$$
\frac{d U_{\mathrm{eff}}}{d r}=0
$$

as $r_{n}=M / 2$. It is interesting that the unstable circular orbits are exact null geodesic solution even the original metric is time dependent. The 'physical radius' $\tilde{r}_{n}=\tilde{M} / 2=\sqrt{t_{1} / t_{0}} M / 2$ increases in time, and $\tilde{r}_{n}<\tilde{r}_{\mathrm{qISCO}}$. The frequency of the photon received by a distant observer, whose proper time is $t$, is red shifted by the scale factor $a(t)$. 


\section{DISCUSSIONS TOWARDS STABILITY ANALYSIS}

Since the present metric (2) describes a charged non-extremal black string, one may expect that the spacetime has the Gregory-Laflamme instability [53]. On the other hand, since the direction of an extra dimension is shrinking as time increases in the solution, one may also think that the spacetime will be stabilized at late time since the compactified extra dimension $w$ prohibits long wave length modes of perturbation. We consider that the stability analysis for this spacetime is interesting, but it is hard to study it since the background spacetime is time-dependent. In this section, towards the stability analysis, we give some discussions from two points of view. One is that by focusing the late time geometry near the horizon which is approximately a static black string geometry, we can study the stability problem on it. The other is to consider the time evolution as a sequence of known static spacetimes with different physical parameters. Interestingly, each discussion suggests an opposite result.

\section{A. Gregory-Laflamme instability for the late time geometry near the horizon}

Near the horizon, the metric behaves approximately that of a static black string solution (20) at late time. In fact, this geometry can be obtained by taking a special limit of the charged black string solution [10] (see Appendix B]). In [54], Frolov and Shoom already showed the existence a Gregory-Laflamme instability for the charged black string solution for general parameters. Surprisingly, the master equation for the zero mode gravitational perturbation only depends on a single parameter while the background spacetime has two parameters, i.e., mass and charge parameters. Thus, we can also expect that the late time geometry near the horizon, which can be obtained by a parameter limit, also has a GregoryLaflamme instability.

We should note that by taking the limit to the late time geometry near the horizon (20) from the charged string solution with two parameters, the asymptotic structure is also drastically changed as shown in Appendix. B. Before taking the limit, the spacetime is asymptotically flat towards the perpendicular direction to the black string, but after the limit, $t-r$ part of the metric asymptotes to $\mathrm{AdS}_{2}$ and the size of extra dimension at infinity becomes zero. So, we should carefully check whether the boundary conditions and the 
gauge conditions used in [54] are still physically reasonable or not. Fortunately, as shown in Appendix C, such the difference in asymptotic structure does not affect on the derivation of the master equation and the boundary condition of it formally. Thus, we can say that the late time geometry near the horizon (2) also has a Gregory-Laflamme mode whose critical wave number along $w$ direction is given by

$$
k_{\mathrm{cr}}=0.876 \cdots,
$$

as like [54].

\section{B. Time evolution as a sequence of static spacetimes}

Let us focus on the geometry near the time slice $t=t_{1}=$ const., the metric (2) behaves

$$
d s^{2}=-\left(1+\frac{M}{r}\right)^{-2} d t^{2}+\left(1+\frac{M}{r}\right)\left[a\left(t_{1}\right)^{2}\left(d r^{2}+r^{2} d \Omega_{\mathrm{S}^{2}}^{2}\right)+\frac{1}{a\left(t_{1}\right)^{2}} d w^{2}\right] .
$$

Using the radial coordinate $\tilde{r}=a\left(t_{1}\right) r$, we find

$$
d s^{2}=-\left(1+\frac{\tilde{M}}{\tilde{r}}\right)^{-2} d t^{2}+\left(1+\frac{\tilde{M}}{\tilde{r}}\right)\left[d \tilde{r}^{2}+\tilde{r}^{2} d \Omega_{\mathrm{S}^{2}}^{2}+\frac{1}{a\left(t_{1}\right)^{2}} d w^{2}\right]
$$

This approximate geometry can be obtained by taking an extremal limit of the charged black string solution [10] (see Appendix B). In the extremal limit, the mass parameter is $\tilde{M}$ and the size of extra dimension is $w_{0} / a$ if $w$ direction is compactified with the period $w_{0}$. We can consider that the time evolution is approximately described by increasing the scale factor $a$. If the time scale of the instability is shorter than the Hubble scale, we can expect that such a physical phenomenon is well described in this spacetime (58). From this point of view, the mass parameter increases and the size of the extra dimension decreases as time increases. This suggests that the spacetime will be stabilized at late time. ${ }^{8}$

\footnotetext{
8 In fact, the extremal limit of the charged black string contains a curvature singularity on the horizon (see Appendix (B). However, we can still use the result in [54] as far as we consider a boundary condition where the perturbed quantiles do not diverge at the horizon and infinity. We consider that such a boundary condition is physically reasonable now, since our original time-dependent solution (2) is regular on the horizon.
} 


\section{SUMMARY AND DISCUSSION}

We have investigated charged black string solutions residing in a five-dimensional Kasner universe where spatial three dimensions expand and an extra dimension contracts. The spacetime has an initial spatial singularity and admits an analytic extension across the event horizon. The inner region is described by a time reversal of the outer region. We can also generalize our solution to multi-black string and multi-black hole system. In this system, the event horizon of a black string is still analytic while both time-dependent and higher-dimensional multi black objects usually have non-smooth event horizon [9, 48, 55 58].

Although there is no exact timelike Killing vector in the spacetime, the geometry is approximately static near the horizon. In fact, the spacetime admits a unique second order asymptotic Killing generator which satisfies an approximate Killing equation. We can also see that the event horizon is an isolated horizon [51]. The late time geometry near the horizon rapidly approaches the geometry of a static exact charged black string solution.

We have studied motions of test particles in the spacetime and seen that quasi stable circular orbits exist. If the time scale of cosmological evolution is much longer than that of orbiting particle near the black string, we can effectively consider the scale factor to be constant during its typical motion. From this point of view, we analyze the effective potential and show the existence of quasi circular orbits and quasi ISCO. The radius of a quasi circular orbit is slowly decreasing with time evolution, then its orbit becomes an inspiral orbit. This is because the energy of particle is not conserved due to the cosmological expansion. After the particle reaches the quasi-ISCO radius, then the particle plunges into the horizon immediately. If we focus on massless particles with $p_{w}=0$, equation of motion reduces to that on a static black hole since the spacetime is effectively conformal static. We have shown that unstable circular orbits of photon exist as exact solutions.

We have given short discussions towards stability analysis from two approximate points of view. One is that by focusing the late time geometry near the horizon which is approximately the geometry of a static charged black string solution, we can discuss the stability problem. This analysis suggests the existence of Gregory-Laflamme instability at late time. On the other hand, since the direction of an extra dimension is shrinking, we can also expect that the spacetime will be stabilized at late time since the compactified extra dimension $w$ prohibits

long wave length modes of perturbation. By considering the time evolution as a sequence 
of static spacetime, we have obtained a suggestion that the spacetime will be stabilized at late time. Since each discussion suggests an opposite result, the stability analysis of our spacetime without approximation is an interesting open question. However, this is not an easy task because we need to study the perturbation around time dependent spacetime. We leave this problem for future work.

Note added: D. Klemm and M. Nozawa study black holes in the expanding universe from the dimensional reduction of supersymmetric solutions in (un)gauged supergravities [59]. They show that our solution can be transformed, via the four-dimensional electromagnetic duality, into a supersymmetric solution to the five-dimensional minimal ungauged supergravity.

\section{Acknowledgments}

We thank D. Klemm and M. Nozawa for reading the manuscript and sharing information

before the submission. We would like to thank G. Gibbons, E. Gourgoulhon, T. Harada, T. Houri, H. Kodama, K.-i. Nakao, and B. Way for fruitful comments. This work is supported by the Grant-in-Aid for Scientific Research No.24540282. M.K. is supported by a grant for research abroad from JSPS.

\section{Appendix A: Case of multi-black holes/strings}

The solution (2) can be easily generalized to multi-black string and multi-black hole solutions. The metric and the Maxwell field of such solutions are given by

$$
\begin{aligned}
d s^{2} & =-H^{-2} d t^{2}+H\left[V\left(d x^{2}+d y^{2}+d z^{2}\right)+V^{-1}(d w+\boldsymbol{\omega})^{2}\right] \\
A_{\mu} d x^{\mu} & = \pm \frac{\sqrt{3}}{2} H^{-1} d t
\end{aligned}
$$

where

$$
\begin{aligned}
H & =1+\sum_{i} \frac{M_{i}}{\left|\boldsymbol{x}-\boldsymbol{x}_{i}\right|}, \\
V(t, r) & =\frac{t}{t_{0}}+\sum_{i} \frac{N_{i}}{\left|\boldsymbol{x}-\boldsymbol{x}_{i}\right|},
\end{aligned}
$$


the 1 -form $\boldsymbol{\omega}$ is determined by $\nabla \times \boldsymbol{\omega}=\nabla V$, and $t_{0}, M_{i}, N_{i}$ are non-negative constants, and $\boldsymbol{x}=(x, y, z), \boldsymbol{x}_{i}=\left(x_{i}, y_{i}, z_{i}\right)$ denote a position vector and a constant vector on the three-dimensional flat Euclidean space.

Black string and black hole horizons are located at $t=\infty, \boldsymbol{x}=\boldsymbol{x}_{i}$ with $t\left|\boldsymbol{x}-\boldsymbol{x}_{i}\right|=$ finite. The parameter $N_{i}$ controls the horizon topology of black strings and black holes. A point source $\boldsymbol{x}=\boldsymbol{x}_{i}$ with $M_{i} \neq 0, N_{i}=0$ describes a dynamical black string discussed in the present paper, while a point source $\boldsymbol{x}=\boldsymbol{x}_{i}$ with $M_{i} \neq 0, N_{i} \neq 0$ describes a dynamical black hole discussed in [48]. Here, in the limit $t_{0} \rightarrow \infty$ with $N_{1} \neq 0$, otherwise $N_{i}=0$, the metric (A1) describes the five-dimensional extremal charged static asymptotically flat multi-black holes [25]. On the other hand, introducing the new coordinate $t^{\prime}=t-t_{0}$ then taking the limit $t_{0} \rightarrow \infty$, the solution (A1) reduces to five-dimensional static Kaluza-Klein multi-black hole solutions [29].

In the following, we show that the multi-black string solutions still admit analytic extensions across the event horizons of the black strings.

\section{Extension across the event horizon in multi-black string system}

For simplicity, we restrict ourselves to the cases of two black strings, i.e., $M_{1} \neq 0, M_{2} \neq 0$ and $N_{1}=N_{2}=0$, otherwise $M_{i}=0, N_{i}=0$. Without loss of generality, we can put the locations of two point sources as $\boldsymbol{x}_{1}=(0,0,0)$ and $\boldsymbol{x}_{2}=(0,0, \delta)$, where the constant $\delta$ denotes the separation between two black strings. In this case, the metric and the Maxwell field are

$$
\begin{aligned}
d s^{2} & =-H(r, \theta)^{-2} d t^{2}+H(r, \theta)\left[\frac{t}{t_{0}}\left(d r^{2}+r^{2} d \Omega_{\mathrm{S}^{2}}^{2}\right)+\frac{t_{0}}{t} d w^{2}\right], \\
A_{\mu} d x^{\mu} & = \pm \frac{\sqrt{3}}{2} H(r, \theta)^{-1} d t
\end{aligned}
$$

where $H$ is given by

$$
H(r, \theta)=1+\frac{M_{1}}{r}+\frac{M_{2}}{\sqrt{r^{2}+\delta^{2}-2 \delta r \cos \theta}} .
$$

Similar to the single black string case, to extend the metric (A5) across the surface $r=0, t=\infty$ with $r t=$ const., we consider the null geodesics near the surface. The null condition is given by

$$
-H(r, \theta)^{-2} d t^{2}+H(r, \theta) \frac{t}{t_{0}} d r^{2}=0 .
$$


Since, near $r=0$, the function $H$ behaves as

$$
H \simeq \frac{M_{1}}{r}+1+\frac{M_{2}}{\delta}
$$

then we have

$$
\left(\frac{d t}{d r}\right)^{2}=\frac{t}{t_{0}}\left(\frac{M_{1}}{r}+1+\frac{M_{2}}{\delta}\right)^{3} .
$$

To obtain coordinates across the surface $r=0, t=\infty$ with $r t=$ const., we use an approximate ingoing future null geodesics near $r=0$

$$
t r=\frac{1}{4 M_{1} t_{0}}\left[2 M_{1}^{2}+u \sqrt{r M_{1}\left(1+\frac{M_{2}}{\delta}\right)}-3 r M_{1}\left(1+\frac{M_{2}}{\delta}\right)\right]^{2},
$$

where $u$ denotes an arbitrary parameter which classifies the approximate null geodesics.

Using the curves (A11), we introduce new coordinates $(u, \rho)$ as

$$
\begin{aligned}
r & =\frac{\delta \rho^{2}}{M_{1}\left(M_{2}+\delta\right)}, \\
t & =\frac{\left(M_{2}+\delta\right)\left(2 M_{1}^{2}+\rho(u-3 \rho)\right)^{2}}{4 t_{0} \delta \rho^{2}},
\end{aligned}
$$

then we rewrite the metric (A5) and the Maxwell field ( $\underline{\mathrm{A} 6})$ in the $(u, \rho)$ coordinates as

$$
\begin{aligned}
d s^{2}= & \frac{\left(M_{2}+\delta\right)^{2}\left(2 M_{1}^{2}+\rho(u-3 \rho)\right)^{2}}{4 t_{0}^{2} \delta^{2} \rho^{4} \tilde{H}^{2}}\left[-\rho^{2} d u^{2}+\rho^{4}\left(\frac{4 \delta^{3} \tilde{H}^{3}}{M_{1}^{2}\left(M_{2}+\delta\right)^{3}}-\frac{\left(2 M_{1}^{2}+3 \rho^{2}\right)^{2}}{\rho^{6}}\right) d \rho^{2}\right. \\
& \left.+2\left(2 M_{1}^{2}+3 \rho^{2}\right) d u d \rho+\frac{\delta^{3} \rho^{6} \tilde{H}^{3}}{M_{1}^{2}\left(M_{2}+\delta\right)^{3}} d \Omega_{\mathrm{S}^{2}}^{2}+\frac{16 t_{0}^{4} \delta^{3} \rho^{6} \tilde{H}^{3}}{\left(M_{2}+\delta\right)^{3}\left(2 M_{1}^{2}+\rho(u-3 \rho)\right)^{4}} d w^{2}\right], \\
A_{\mu} d x^{\mu}= & \pm \frac{\sqrt{3}\left(M_{2}+\delta\right)\left(2 M_{1}^{2}+\rho(u-3 \rho)\right)}{4 t_{0} \delta \rho^{3} \tilde{H}}\left[\rho^{2} d u-\left(2 M_{1}^{2}+3 \rho^{2}\right) d \rho\right],
\end{aligned}
$$

where

$$
\tilde{H}=1+\frac{M_{1}^{2}\left(M_{2}+\delta\right)}{\delta \rho^{2}}+\frac{M_{1} M_{2}\left(M_{2}+\delta\right)}{\delta \sqrt{\rho^{4}+M_{1}^{2}\left(M_{2}+\delta\right)^{2}-2 M_{1}\left(M_{2}+\delta\right) \rho^{2} \cos \theta}} .
$$

We see that the metric (A14) is analytic. When $M_{2}=0$, the metric (A14) and the Maxwell field (A15) reduce to those in the single black string case.

In the limit $\rho \rightarrow 0$ with $u=$ finite (equivalently, $r \rightarrow 0, t \rightarrow \infty$ with $\operatorname{tr}=M_{1}^{3} / t_{0}$ ), we obtain

$$
d s^{2} \rightarrow \frac{4 M_{1}^{2}}{t_{0}^{2}} d u d \rho+\frac{M_{1}^{4}}{t_{0}^{2}} d \Omega_{\mathrm{S}^{2}}^{2}+\frac{t_{0}^{2}}{M_{1}^{2}} d w^{2}
$$


We find that the metric (A14) is regular at the horizon $\rho=0$.

Thus the solutions (A5) with (A7) describe a pair of charged black strings which have analytic event horizons without a singularity on the black string horizons in the five-dimensional Kaluza-Klein universe.

\section{Appendix B: Electrically charged black string with two parameters}

We consider a static charged black string solution of five-dimensional Einstein-Maxwell equations whose metric and gauge 1-form are given by

$$
\begin{aligned}
& d s^{2}=-\left(1-\frac{r_{+}}{r}\right)\left(1-\frac{r_{-}}{r}\right) d t^{2}+\left(1-\frac{r_{+}}{r}\right)^{-1} d r^{2} \\
&+r^{2}\left(1-\frac{r_{-}}{r}\right) d \Omega_{\mathrm{S}^{2}}^{2}+\left(1-\frac{r_{-}}{r}\right)^{-1} d z^{2}, \\
& A_{\mu} d x^{\mu}= \pm \frac{\sqrt{3}}{2} \frac{\sqrt{r_{+} r_{-}}}{r} d t,
\end{aligned}
$$

where $r_{ \pm}$are parameters with the inequality $r_{-} \leq r_{+}$, and the coordinate range outside the horizon is $r_{+}<r<\infty$. The event horizon locates at $r=r_{+}$and the curvature singularity locates at $r=r_{-}$. This solution is a special limit of the diatonic charged black string

solution constructed by Bleyer and Ivashchuk [10]. Physical properties of the solution (B1) were studied in [11, 54].

First, we consider the extremal limit of this solution, i.e., $r_{-} \rightarrow r_{+}$. By introducing coordinates $\tilde{r}:=r-r_{+}$and $w=a z$, we see that the metric (B1) takes the form of (58). Note that a curvature singularity locates at the horizon $r=r_{+}\left(=r_{-}\right)$in this extremal case.

Second, we consider a special limit to obtain the late time geometry near the horizon. Let us introduce new coordinates

$$
T:=\frac{R_{h}^{2}}{r_{-} \sqrt{r_{-}^{2}-R_{h}^{2}}} t, \quad R^{2}:=r_{-}^{2}\left(1-\frac{r_{-}}{r}\right), \quad W:=\frac{r_{-} z}{R_{h}},
$$

with $R_{h}^{2}:=r_{-}^{2}\left(1-r_{-} / r_{+}\right)$, then the metric (B1) becomes

$$
d s^{2}=-\frac{R^{2}\left(R^{2}-R_{h}^{2}\right)}{R_{h}^{4}} d T^{2}+\frac{4 R^{2}}{R^{2}-R_{h}^{2}} \frac{1-R_{h}^{2} / r_{-}^{2}}{\left(1-R^{2} / r_{-}^{2}\right)^{4}} d R^{2}+\frac{R^{2}}{\left(1-R^{2} / r_{-}^{2}\right)^{2}} d \Omega_{\mathrm{S}^{2}}^{2}+\frac{R_{h}^{2}}{R^{2}} d W^{2},
$$

where the coordinate range outside the horizon is $R_{h}<R<r_{-}$. If we take a limit $r_{-} \rightarrow$ $\infty$ while keeping $R_{h}=$ finite for this metric, we obtain the late time geometry near the 
horizon (20). In this limit, we can see that $T-R$ part of the metric asymptotes to $\mathrm{AdS}_{2}$ near the infinity and the size of the extra dimension at infinity is zero. We show the Penrose diagrams for these static charged black strings in Fig. 6 ,
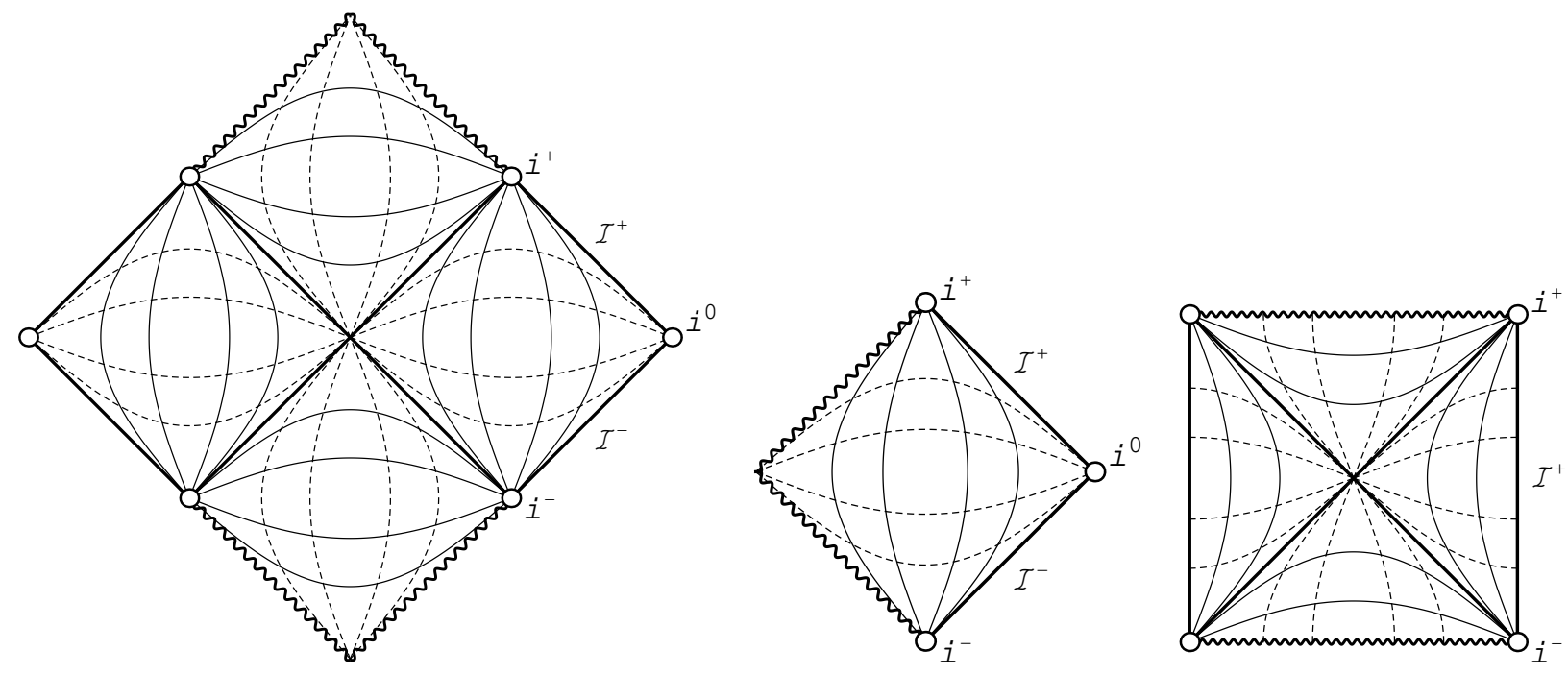

FIG. 6: Penrose diagrams of maximal extensions for static charged black strings. Each figure corresponds to charged black strings with two parameters (left), extremal limit (center), $r_{-} \rightarrow \infty$ limit with the metric (B4) (right), respectively. The wavy lines are curvature singularities. Dashed curves denote $t=$ const. (left, center) or $T=$ const. (right) surfaces, and thin solid curves denote $r=$ const. (left, center) or $R=$ const. (right) surfaces.

Appendix C: Master equation for zero mode in the late time geometry near the horizon

In ref. [54], it is shown that gravitational and electric zero-mode perturbations are decoupled for the static charged black string solution (B1), and only gravitational perturbation has unstable modes. Since it is expected that the late time geometry near the horizon has a similar property, we take the same ansatz for the metric and Maxwell field as [54],

$$
\begin{aligned}
d s^{2}= & -\frac{R^{2}\left(R^{2}-R_{h}^{2}\right)}{R_{h}^{4}} e^{2 \tau(R, W)} d T^{2}+\frac{4 R^{2}}{R^{2}-R_{h}^{2}} e^{2 \sigma(R, W)} d R^{2}+R^{2} e^{2 \gamma(R, W)} d \Omega_{\mathrm{S}^{2}}^{2} \\
& +\frac{R_{h}^{2}}{R^{2}} e^{2 \beta(R, W)}(d W-\alpha(R, W) d R)^{2}, \\
A_{\mu} d x^{\mu}= & \pm \frac{\sqrt{3}}{2} R^{2} d T .
\end{aligned}
$$


By taking the first order of the small variables $\tau(R, W), \beta(R, W), \sigma(R, W), \gamma(R, W), \alpha(R, W)$ for Einstein-Maxwell equations, we can obtain the linearized equations.

Let us consider the Fourier transformation for $W$ direction,

$$
\begin{aligned}
& \tau(R, W)=\tilde{\tau}(R) e^{i k W}, \quad \sigma(R, W)=\tilde{\sigma}(R) e^{i k W}, \quad \gamma(R, W)=\tilde{\gamma}(R) e^{i k W}, \\
& \alpha(R, W)=\tilde{\alpha}(R) e^{i k W} \quad \beta(R, W)=\tilde{\beta}(R) e^{i k W} .
\end{aligned}
$$

By using the gauge transformations $g_{\mu \nu} \rightarrow g_{\mu \nu}-2 \nabla_{(\mu} \xi_{\nu)}$, the perturbed quantities behave as

$$
\begin{aligned}
& \tilde{\tau}(R) \rightarrow \tilde{\tau}(R)-\frac{2 R^{2}-R_{h}^{2}}{4 R^{3}} \tilde{\xi}_{R}(R), \\
& \tilde{\sigma}(R) \rightarrow \tilde{\sigma}(R)-\frac{R_{h}^{2}}{4 R^{3}} \tilde{\xi}_{R}(R)-\frac{R\left(R^{2}-R_{h}^{2}\right)}{4 R^{3}} \tilde{\xi}_{R}^{\prime}(R), \\
& \tilde{\gamma}(R) \rightarrow \tilde{\gamma}(R)-\frac{R^{2}-R_{h}^{2}}{4 R^{3}} \tilde{\xi}_{R}(R), \\
& \tilde{\alpha}(R) \rightarrow \tilde{\alpha}(R)+i k R^{2} \tilde{\xi}_{R}(R)+2 R \tilde{\xi}_{W}(R)+R^{2} \tilde{\xi}_{W}^{\prime}(R), \\
& \tilde{\beta}(R) \rightarrow \tilde{\beta}(R)+\frac{R^{2}-R_{h}^{2}}{4 R^{3}} \tilde{\xi}_{R}(R)-i k R^{2} \tilde{\xi}_{W}(R) .
\end{aligned}
$$

We can choose the gauge condition

$$
\tilde{\tau}(R)=0, \quad \tilde{\beta}(R)=0 .
$$

Note that this completely fixes the gauge degrees of freedom and we can choose this gauge condition even near the horizon and infinity. ${ }^{9}$ After some calculations, we obtain a single master equation for $\tilde{\gamma}(R)$ as

$$
\frac{R^{2}-R_{h}^{2}}{4 R^{4}} \tilde{\gamma}^{\prime \prime}(R)-\frac{4 R^{4}-3 R^{2} R_{h}^{2}-3 R_{h}^{4}}{4 R^{5}\left(4 R^{2}-3 R_{h}^{2}\right)} \tilde{\gamma}^{\prime}(R)+\frac{2 R_{h}^{2}}{R^{4}\left(4 R^{2}-3 R_{h}^{2}\right)} \tilde{\gamma}(R)=k^{2} \tilde{\gamma}(R),
$$

The relations among $\tilde{\gamma}(R)$ and the other variables are given by

$$
\begin{aligned}
\tilde{\alpha}(R) & =\frac{-2 i\left[4\left(-R R_{h}^{2}+k^{2}\left(4 R^{7}-3 R^{5} R h^{2}\right)\right) \tilde{\gamma}(R)+R_{h}^{2}\left(2 R^{2}-3 R_{h}^{2}\right) \tilde{\gamma}^{\prime}(R)\right]}{k\left(4 R^{2}-3 R_{h}^{2}\right)^{2}}, \\
\tilde{\sigma}(R) & =\frac{2\left(R^{2}-R_{h}^{2}\right)\left(2 \tilde{\gamma}(R)+R \tilde{\gamma}^{\prime}(R)\right)}{4 R^{2}-3 R_{h}^{2}}
\end{aligned}
$$

9 When we consider the gauge transformation (다), $\tilde{\xi}_{R}(R)$ may diverge at most of the order of $R$ near infinity. However linear perturbation is still good approximation. For the condition where the linear perturbation is a good approximation, we should impose that the perturbed quantities $\tilde{\tau}(R), \tilde{\sigma}(R), \tilde{\gamma}(R), \tilde{\alpha}(R), \tilde{\beta}(R)$ take finite value in $R_{h} \leq R \leq \infty$, and the gauge transformation (C8) does not violate this. 
Introducing a new radial coordinate

$$
R^{2}=\frac{R_{h}^{2}}{1-x}
$$

where $x=0$ and $x=1$ correspond to the horizon and spatial infinity, respectively, the master equation becomes

$$
x^{2} \frac{d^{2} \tilde{\gamma}(x)}{d x^{2}}-x \frac{3 x^{2}+6 x-1}{(1-x)(1+3 x)} \frac{d \tilde{\gamma}(x)}{d x}+\left[\frac{2 x}{(1-x)(1+3 x)}-\frac{x}{(1-x)^{4}} k^{2}\right] \tilde{\gamma}(x)=0 .
$$

This completely coincides with the master equation in [54].

The boundary conditions used in [54] are obtained by imposing that the perturbed quantities do not diverge at the horizon and infinity. Althogh the asymptotic structure of (20) is different from that of the static charged black string (B1), the boundary conditions for the master equation (C9) that we should impose are the same, i.e., the perturbed quantities do not diverge at $R=R_{h}$ and $R=\infty$. Since we solve the same equation with the same boundary condition formally, we obtain the same result as [54], i.e., the spacetime is unstable against long wave perturbations whose critical wave number is $k_{\text {cr }}=0.876 \cdots$.

[1] T. Appelquist, A. Chodos and P. G. O. Freund, "Modern Kaluza-Klein Theories," READING, USA: ADDISON-WESLEY (1987) 619 P. (FRONTIERS IN PHYSICS, 65)

[2] A. Chodos and S. L. Detweiler, Phys. Rev. D 21, 2167 (1980).

[3] P. G. O. Freund, Nucl. Phys. B 209, 146 (1982).

[4] S. Randjbar-Daemi, A. Salam and J. A. Strathdee, Phys. Lett. B 135, 388 (1984).

[5] D. Sahdev, Phys. Lett. B 137, 155 (1984).

[6] H. Ishihara, Prog. Theor. Phys. 72, 376 (1984).

[7] H. Ishihara, Phys. Lett. B 179, 217 (1986).

[8] G. T. Horowitz and A. Strominger, Nucl. Phys. B 360, 197 (1991).

[9] G. W. Gibbons, G. T. Horowitz and P. K. Townsend, Class. Quant. Grav. 12, 297 (1995) hep-th/9410073.

[10] U. Bleyer and V. D. Ivashchuk, Phys. Lett. B 332, 292 (1994) gr-qc/9405018.

[11] G. T. Horowitz and K. Maeda, Phys. Rev. D 65, 104028 (2002) hep-th/0201241.

[12] R. Emparan and H. S. Reall, Phys. Rev. Lett. 88, 101101 (2002) hep-th/0110260. 
[13] R. C. Myers and M. J. Perry, Annals Phys. 172, 304 (1986).

[14] R. Emparan and H. S. Reall, Living Rev. Rel. 11, 6 (2008) [arXiv:0801.3471 [hep-th]].

[15] P. Dobiasch and D. Maison, Gen. Rel. Grav. 14, 231 (1982).

[16] G. W. Gibbons and D. L. Wiltshire, Annals Phys. 167, 201 (1986) [Erratum-ibid. 176, 393 (1987)].

[17] H. Ishihara and K. Matsuno, Prog. Theor. Phys. 116, 417 (2006) arXiv:hep-th/0510094.

[18] T. Nakagawa, H. Ishihara, K. Matsuno and S. Tomizawa, Phys. Rev. D 77, 044040 (2008) arXiv:0801.0164 [hep-th]].

[19] S. Tomizawa, H. Ishihara, K. Matsuno and T. Nakagawa, Prog. Theor. Phys. 121, 823 (2009) arXiv:0803.3873 [hep-th]].

[20] K. Matsuno, H. Ishihara, M. Kimura and T. Tatsuoka, Phys. Rev. D 86, 044036 (2012) arXiv:1206.4818 [hep-th]].

[21] S. Tomizawa and S. 'y. Mizoguchi, Phys. Rev. D 87, 024027 (2013) [arXiv:1210.6723 [hep-th]].

[22] C. Stelea, C. Dariescu and M. A. Dariescu, Phys. Rev. D 87, 024039 (2013) arXiv:1211.3154 $[\mathrm{gr}-\mathrm{qc}]]$.

[23] S. D. Majumdar, Phys. Rev. 72, 390 (1947).

[24] A. Papapetrou, Proc. Roy. Irish Acad. (Sect. A) A 51, 191 (1947).

[25] R. C. Myers, Phys. Rev. D 35, 455 (1987).

[26] J. C. Breckenridge, R. C. Myers, A. W. Peet and C. Vafa, Phys. Lett. B 391, 93 (1997) hep-th/9602065.

[27] J. P. Gauntlett, J. B. Gutowski, C. M. Hull, S. Pakis and H. S. Reall, Class. Quant. Grav. 20, 4587 (2003) arXiv:hep-th/0209114.

[28] D. Gaiotto, A. Strominger, X. Yin, JHEP 0602, 024 (2006). hep-th/0503217.

[29] H. Ishihara, M. Kimura, K. Matsuno and S. Tomizawa, Class. Quant. Grav. 23, 6919 (2006) [hep-th/0605030].

[30] H. Ishihara, M. Kimura, K. Matsuno and S. Tomizawa, Phys. Rev. D 74, 047501 (2006) hep-th/0607035.

[31] K. Matsuno, H. Ishihara, T. Nakagawa and S. Tomizawa, Phys. Rev. D 78, 064016 (2008) arXiv:0806.3316 [hep-th]].

[32] T. Tatsuoka, H. Ishihara, M. Kimura and K. Matsuno, Phys. Rev. D 85, 044006 (2012) arXiv:1110.6731 [hep-th]]. 
[33] K. Matsuno, H. Ishihara, M. Kimura and T. Tatsuoka, Phys. Rev. D 86, 104054 (2012) arXiv:1208.5536 [hep-th]].

[34] K. Matsuno, H. Ishihara and M. Kimura, Class. Quant. Grav. 32, no. 21, 215008 (2015) arXiv:1504.04203 [hep-th]].

[35] D. Kastor and J. H. Traschen, Phys. Rev. D 47, 5370 (1993) hep-th/9212035.

[36] S. Chimento and D. Klemm, JHEP 1304, 129 (2013) [arXiv:1212.5494 [hep-th]].

[37] S. Chimento and D. Klemm, Class. Quant. Grav. 32, no. 4, 045006 (2015) arXiv:1405.5343 [hep-th]].

[38] L. A. J. London, Nucl. Phys. B 434, 709 (1995).

[39] D. Klemm and W. A. Sabra, Phys. Lett. B 503, 147 (2001) hep-th/0010200].

[40] H. Ishihara, M. Kimura and S. Tomizawa, Class. Quant. Grav. 23, L89 (2006) hep-th/0609165.

[41] D. Ida, H. Ishihara, M. Kimura, K. Matsuno, Y. Morisawa and S. Tomizawa, Class. Quant. Grav. 24, 3141 (2007) hep-th/0702148 [HEP-TH]].

[42] K. Matsuno, H. Ishihara, M. Kimura and S. Tomizawa, Phys. Rev. D 76, 104037 (2007) arXiv:0707.1757 [hep-th]].

[43] M. Kimura, Phys. Rev. D 80, 044012 (2009) [arXiv:0904.4311 [gr-qc]].

[44] G. W. Gibbons, H. Lu and C. N. Pope, Phys. Rev. Lett. 94, 131602 (2005) hep-th/0501117.

[45] K. -i. Maeda, N. Ohta and K. Uzawa, JHEP 0906, 051 (2009) arXiv:0903.5483 [hep-th]].

[46] K. -i. Maeda and M. Nozawa, Phys. Rev. D 81, 044017 (2010) arXiv:0912.2811 [hep-th]].

[47] K. -i. Maeda and M. Nozawa, Phys. Rev. D 81, 124038 (2010) arXiv:1003.2849 [gr-qc]].

[48] Y. Kanou, H. Ishihara, M. Kimura, K. Matsuno and T. Tatsuoka, Phys. Rev. D 90, no. 8, 084004 (2014) arXiv:1408.2956 [hep-th]].

[49] J. i. Koga, Phys. Rev. D 64, 124012 (2001) gr-qc/0107096.

[50] J. i. Koga, Class. Quant. Grav. 24, 3067 (2007) gr-qc/0604054.

[51] A. Ashtekar and B. Krishnan, Living Rev. Rel. 7, 10 (2004) gr-qc/0407042.

[52] H. Ishihara, A. Tomimatsu and M. Den, Prog. Theor. Phys. 74, 1045 (1985).

[53] R. Gregory and R. Laflamme, Phys. Rev. Lett. 70, 2837 (1993) hep-th/9301052.

[54] V. P. Frolov and A. A. Shoom, Phys. Rev. D 79, 104002 (2009) arXiv:0903.2893 [hep-th]].

[55] D. R. Brill, G. T. Horowitz, D. Kastor and J. H. Traschen, Phys. Rev. D 49, 840 (1994) gr-qc/9307014. 
[56] D. L. Welch, Phys. Rev. D 52, 985 (1995) hep-th/9502146.

[57] G. N. Candlish and H. S. Reall, Class. Quant. Grav. 24, 6025 (2007) [arXiv:0707.4420 [gr-qc]].

[58] M. Kimura, H. Ishihara, K. Matsuno and T. Tanaka, Class. Quant. Grav. 32, no. 1, 015005 (2015) arXiv:1407.6224 [gr-qc]].

[59] D. Klemm and M. Nozawa, arXiv:1511.01949 [hep-th]. 